\title{
Transmastoid Approach for Retrolabyrinthine and Translabyrinthine
}

\author{
Tetsuro Sameshima
}

\subsection{Introduction}

It is very important to understand the anatomical landmark of this approach (Fig. 8.1). There are some well-known triangle and skin incisions related to retrolabyrinthine and translabyrinthine approaches (Fig. 8.1a, b).

The retrolabyrinthine approach exposes the posterior fossa dura between sigmoid sinus, posterior semicircular canal, jugular bulb, and superior petrosal sinus. Retrolabyrinthine access to the cerebellopontine angle (CPA) with hearing preservation can be accomplished; however, exposure is generally limited. The most frequent indication for an isolated retrolabyrinthine approach is selective vestibular nerve section. More frequently the retrolabyrinthine approach is combined with cutting temporal bone and splitting tentorium to expose lateral pons and basilar artery from the confluence of the vertebral arteries to the dorsum sellae (the combined petrosal approach).

When hearing that preservation is not a consideration, the CPA can be more widely exposed by removing the vestibular labyrinth and skeletonizing the internal auditory canal through the

T. Sameshima

Department of Neurosurgery,

Hamamatsu University School of Medicine,

University Hospital,

Hamamatsu city, Japan translabyrinthine approach. Advocates of this approach cite early identification of the facial nerve in the IAC and minimal brain retraction as factors for improved results in acoustic tumor surgery. Either the retro- or translabyrinthine approaches can be combined with other lateral skull base procedures to enhance exposure, depending on size and location of lesion being exposed and the patient's hearing status such as the total petrosectomy approach with facial nerve translocation.

\subsection{Steps of the Surgery}

\subsubsection{Positioning, Incision, and Bony Landmarks}

The head is held with lateral position, facing away from the surgeon. A postauricular incision through the galea is made, one inch behind the postauricular crease. The incision extends from the mastoid tip and curves forward to end just above the pinna (or midpoint of supramastoid crest) (Fig. 8.1d left).

The scalp is elevated by sharply dissecting the subgaleal connective tissue which spans the galea and the underlying pericranium. The pericranium is contiguous with the temporalis fascia above and the fascia overlying the sternocleidomastoid muscle below. A second incision is made in this deep layer composed of temporalis fascia and 

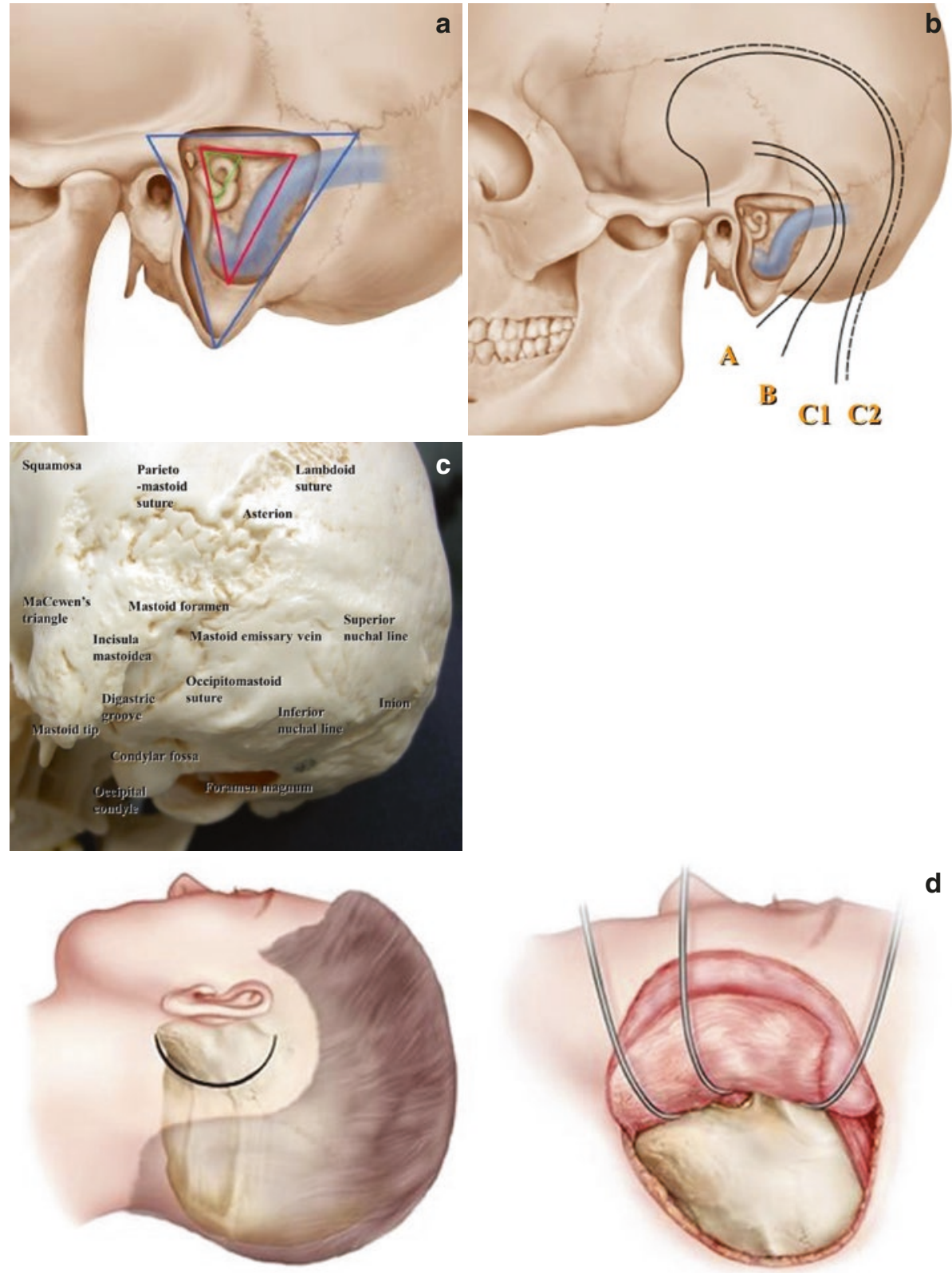

Fig. 8.1 (a) Surface anatomy of the temporal bone and mastoid triangles. Blue, Fukushima's outer mastoid triangle (asterion-root of zygoma posterior point-mastoid tip) [1, 2]; red, Fukushima's inner mastoid triangle (sinodural angleaditus-digastric ridge) [1, 2]; Trautman's triangle (sinodural angle-superior aspect of posterior semicircular canal-jugular bulb) [3]; green, MacEwen's triangle or suprameatal triangle (mastoid antrum) which is a flat or depressive area [4]. (b) Skin incision. (A) Small C-shaped transmastoid incision represents standard transmastoid, retrolabyrinthine, or trans-

labyrinthine incision used by neuro-otologists; (B) extended transmastoid incision represents a combined transmastoid and suboccipital (retrosigmoid) approach frequently used for the preservation of hearing function in acoustic neuroma surgery; (C) combined transpetrosal incision represents a question mark $(\mathrm{C} 1)$ or L-shaped incision (C2) used in this skull base dissection for combined petrosal approach. (c) Bony landmark. (d) Left, skin incision; right, after the skin and muscle elevated and exposing the bony landmarks 
muscle, periosteum, and sternocleidomastoid fascia to fashion a musculofascial flap that is important in obtaining a water tight, cosmetic closure. The two flaps are elevated anteriorly to reveal posterior edge of external auditory canal, spine of Henle, and root of zygoma posterior point. Large blunt scalp hooks are used to reflect these flaps. The bony landmarks which should be visualized at this point are the root of zygoma posterior point, the spine of Henle, the squamosal suture, the asterion, the supramastoid or temporal crest, the mastoid tip, and the digastric groove (Fig. 8.1c, d right).

\subsubsection{Mastoidectomy and Retrolabyrinthine Exposure}

The second step is performance of a mastoidectomy. Using large cutting drill $(5 \sim 6 \mathrm{~mm})$ with continuous irrigation and suction, cortex over the mastoid bone is removed. It is helpful to first outline the boundaries of the bone to be removed using the drill. The anterior border is a slightly curved line, extending from the top of the external auditory meatus to the mastoid tip. The superior margin is along a line roughly perpendicular to the first, extending from the root of zygoma posteriorly to the region of the asterion. These two lines form a skewed " $T$ " that defines the anterior and superior margins of the mastoidectomy (Fig. 8.2a). The junction of these two lines generally marks the surface projection of the region of the mastoid antrum and lateral semicircular canal.

The bone cortex is removed within the boundaries of these lines, working anterior to posterior and superior to inferior. After drilling the cortical bone, air cells are encountered. Posteriorly, over sigmoid sinus, the bone will remain compact. In order to provide maximum exposure, wide cortical removal with cauterization should be performed prior to deeper penetration. Gentle, brushlike strokes with the drill will reveal the compact bone of the sigmoid sinus. Bone removal proceeds, $1 \mathrm{~cm}$ behind the sigmoid, maintaining a uniform depth as the sigmoid is exposed. When the sigmoid has been skeletonized, the mastoid air cells are drilled away to expose the temporal base dura (temporal tegmen) (Fig. 8.2b).

Moving anteriorly, the air cells will be removed to expose the compact bone of the bony labyrinth or the solid angle. The key landmark in this area is the mastoid antrum (Fig. 8.2c-e). This open space defines the anterior limit of bony removal and locates the lateral semicircular canal. Maintaining the same relative depth, air

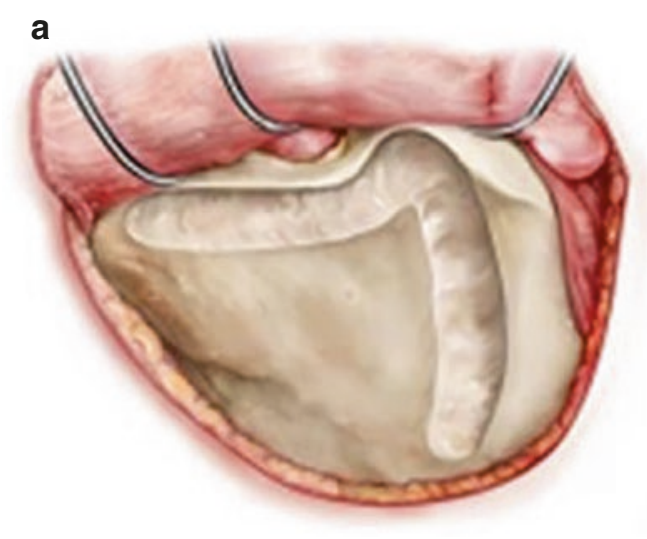

Fig. 8.2 (a) (Left) The anterior and superior margins of the mastoidectomy; (b) (right) the mastoid air cells are removed anteriorly and superiorly to expose the middle fossa dura (temporal tegmen). (c-e) Exposing the mastoid antrum. (f, g) Exposing the semicircular canal and the

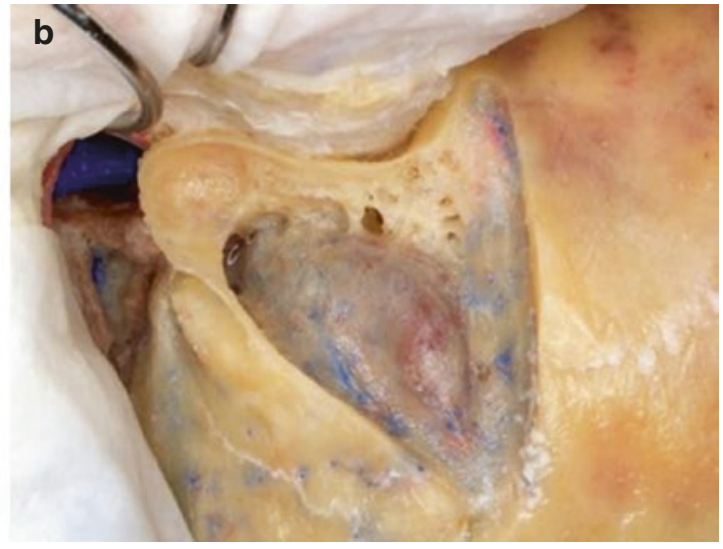

transverse sigmoid sinus. Cadaver (left) and drawing (right). (h) Skeletonizing of the sigmoid sinus, jugular bulb, the fallopian canal, and the entire course of the facial nerve through the mastoid bone 

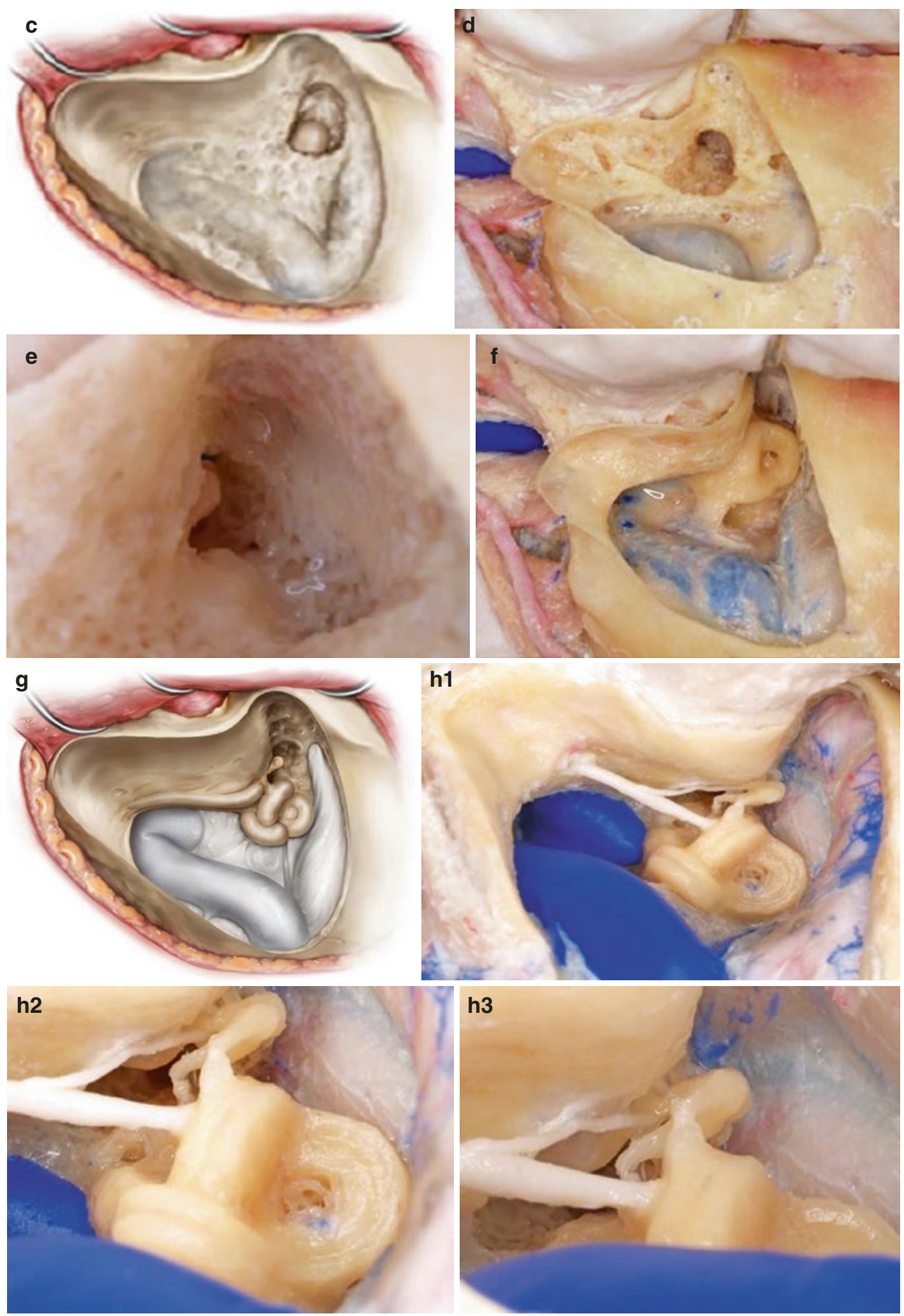

Fig. 8.2 (continued) 
cell removal proceeds inferiorly toward the jugular bulb. After the air cells are drilled away, we will expose digastric ridge. Digastric groove defines the exit of facial nerve from the fallopian canal through the stylomastoid foramen. The stylomastoid foramen lies just medial to anterior edge of digastric ridge. At this point, middle fossa dura, presigmoid dura, and the sinodural angle should be skeletonized. Again, the technique of removing the bone to the point of leaving a thin shell which may be removed with a dissector is practiced to avoid damage to the dura and the venous structures. For maximal exposure in the retrolabyrinthine approach, the posterolateral portion of the bony part of labyrinth must be completely identified. Using medium diamond drill $(2-3 \mathrm{~mm})$, small air cells surrounding the labyrinth are removed. The ridge covering the lateral semicircular canal is first identified as the antrum is opened. The facial nerve will be located parallel and 1-2 $\mathrm{mm}$ in front of lateral semicircular canal at this point. Moving posteriorly, the posterior semicircular canal will be defined (Fig. 8.2f, g). Inferior to posterior semicircular canal, toward the jugular dome, lie the retrofacial air cells. They are drilled away to skeletonize jugular bulb. The dura is incised corresponding the presigmoid region and superior petrosal sinus. The dura is retracted anteriorly exposing CN VII and VIII in the CPA. Frequently the lower cranial nerves can also be visualized.

Anteriorly, approximately $12-15 \mathrm{~mm}$ medial to meatus acusticus external lies the fallopian canal. Therefore, bone removal in the anterior direction at this level must be done with extreme care to avoid violating the fallopian canal. Facial nerve, which lies anterior to labyrinthine structures, is carefully approached, again using the lateral semicircular canal as a landmark. The $\mathrm{CN}$ VII is skeletonized by using the diamond drill started at external genu inferiorly to stylomastoid foramen. Leave a thin layer of the bone around the facial nerve for protection. The drilling must be done with copious and constant irrigation to dissipate heat from the drill.

At this stage certain goals of bone removal should have been achieved, such as exposure of sigmoid sinus and jugular bulb. Exposure of pre- sigmoid dura and middle fossa dura defines the lateral bony labyrinth, clearly visualizing the lateral semicircular canal (LSC) and posterior semicircular canals (PSC) and skeletonizing fallopian canal and exposing the entire facial nerve (Fig. 8.2h).

\subsubsection{Translabyrinthine Drilling/IAC}

The lateral and posterior semicircular canals (PSC) are first opened with the drill. The amputated, or anterior, end of LSC is carefully removed, bearing in mind close relationship of tympanic portion of facial nerve. Preservation of anterior wall of LSC will protect tympanic segment of VII nerve. Removal of superior segment of the posterior semicircular canal will expose the common crus which it shares with the SSC. The SSC is also opened by drilling superiorly and anteriorly. The amputated, or inferior, limb of the PSC is followed until the vestibule. Drilling in this area, lateral and inferior to the vestibule, will expose the vestibular aqueduct as it courses laterally toward the endolymphatic sac. The vestibule is now opened by continuing to remove the bone, following the common crus (Fig. 8.3a, b).

The wall of the vestibule which separates it from the internal auditory canal is only one very thin layer of the bone. The compact bone surrounding the internal auditory canal is identified by drilling along the canal at its superior and inferior edge. It is important in terms of maximizing the exposure, to remove the bone around the canal such that greater than one-half of the circumference of the canal is skeletonized. It is important to remove the bone superiorly and inferiorly so that the anterior-most extent of the canal is accessible. Bone removal inferior to the canal will in some cases expose the cochlear canaliculus which communicates with the CSF and perilymphatic spaces.

The bone around the internal auditory canal is then carefully removed. Beginning in the region of the porus acusticus, the compact bone surrounding the canal is thinned with a small diamond burr until only a thin, repressible shell remains. As the 
drilling proceeds laterally, bear in mind that dura only covers the canal contents for approximately two-thirds of the canal's length. As the bone is thinned at the lateral end of the canal, we will see transverse crest (thin bone) that lies between superior and inferior vestibular nerves. The paper-thin shell of the bone in the region of the porus acusticus is removed first with a fine dissector, with the bone over the lateralmost end canal saved for last. The superior lip of porus is generally the most difficult to manage because of the very close proximity of the facial nerve (Fig. 8.3c-e).
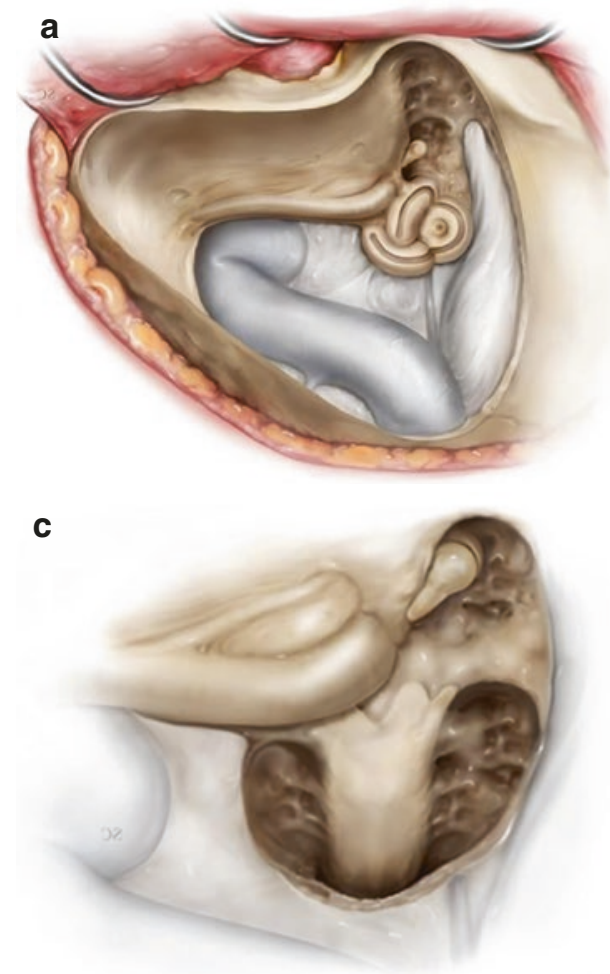

Fig. $8.3(\mathbf{a}, \mathbf{b})$. The vestibule is now opened by continuing to remove the bone, following the common crus. A (left) the drawing, B (right) the cadaver specimen. (c-e) The paper-thin shell of the bone in the region of the porus acusticus is removed first with a fine dissector, with the bone over the lateralmost end of the internal auditory canal saved for last. The superior lip of the porus is gener-
The dura is incised beginning just medial to the sigmoid sinus, 5-10 $\mathrm{mm}$ below superior petrosal sinus and continued in a line toward the midportion of the canal. At region of the porus, dural incision is extended superiorly and inferiorly. Using a \#11 blade, the dura of the canal is cut to expose inferior and superior vestibular nerves. Sectioning of the nerves and then reflecting them laterally will reveal the cochlear and facial nerves. The latter two are separated from the superior and inferior vestibular nerves by Bill's bar at the lateral end (Fig. 8.3f, g).
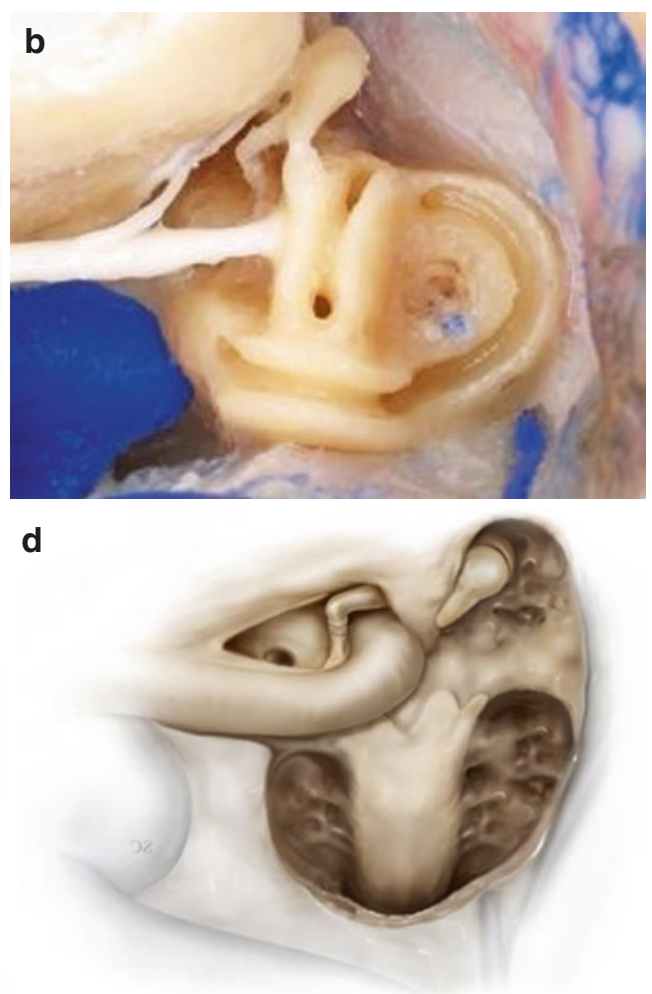

ally the most difficult to manage because of the very close proximity of the facial nerve. (f, g) Sectioning of the nerves and then reflecting them laterally will reveal the cochlear and facial nerves. The latter two are separated from the superior and inferior vestibular nerves by Bill's bar at the lateral end ( $F$ facial nerve, $C$ cochlear nerve, $S V$ superior vestibular nerve, $I V$ inferior vestibular nerve) 

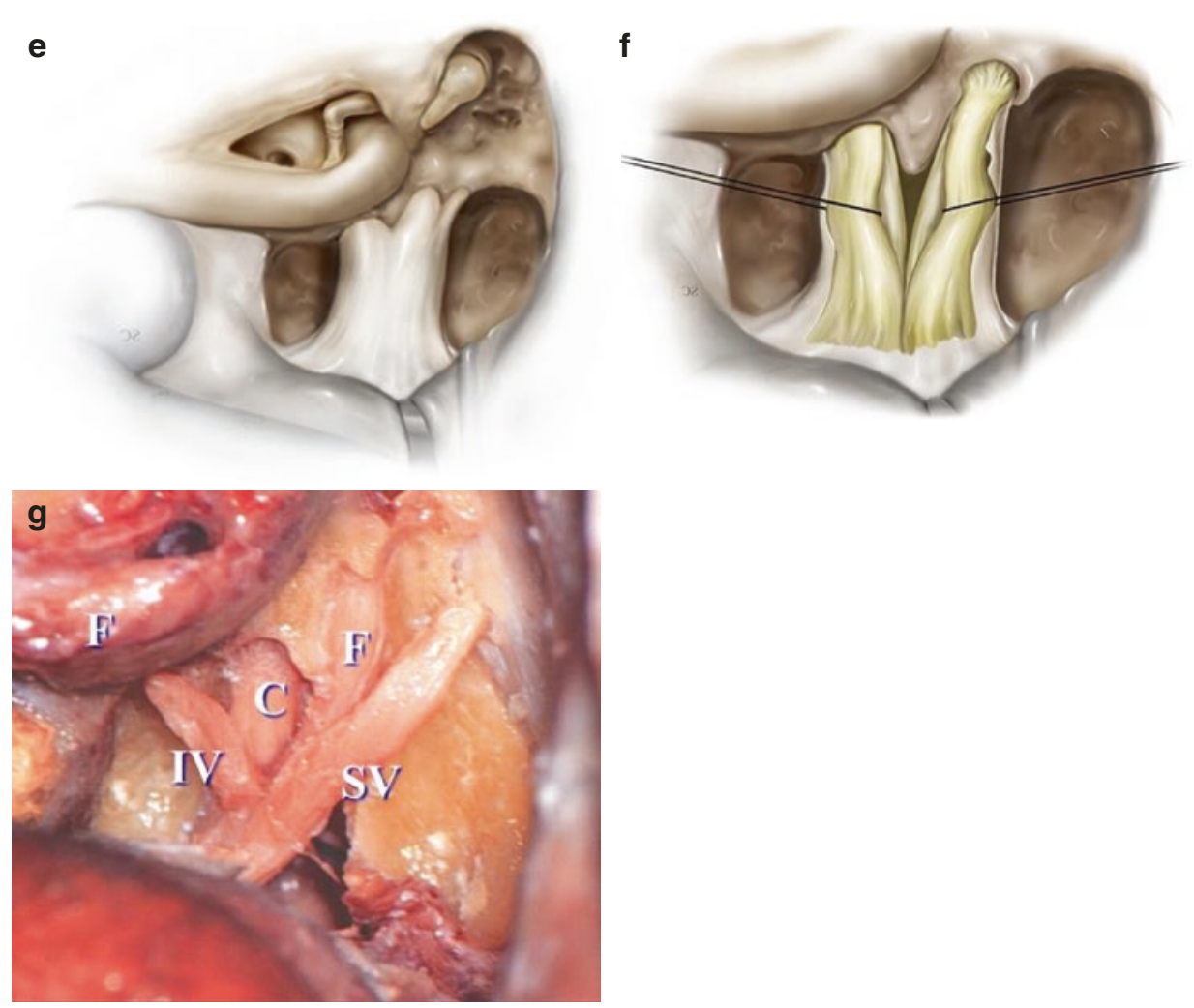

Fig. 8.3 (continued)

The wound is closed in the following manner. The incus is removed, and a piece of temporalis muscle is harvested and placed carefully through the epitympanum occluding the origin of the Eustachian tube. The removal of the incus and obliteration of the Eustachian tube entrance by the muscle reduces the possibility of CSF leakage. The dural incision is closed up to the canal, and carpets of autologous (abdominal) fat are positioned in the gaps of the dura so as to seal the CSF space. The previously fashioned musculofascial flap is closed tightly over the adipose graft, and the skin cut is closed in two layers.

\subsubsection{Combined Transpetrosal Approach}

The head is positioned (Fig. 8.4a) with the option for skin incision. Using the virtual computer image, it shows the transverse sigmoid junction until jugular vein, in the relationship with the labyrinth and its exposure that can be achieved with this approach (Fig. 8.4b). The next illustration (Fig. 8.4c) shows that dural opening, the neurovascular structure. Be aware that the dural opening is parallel to GSPN at the temporal skull base. 

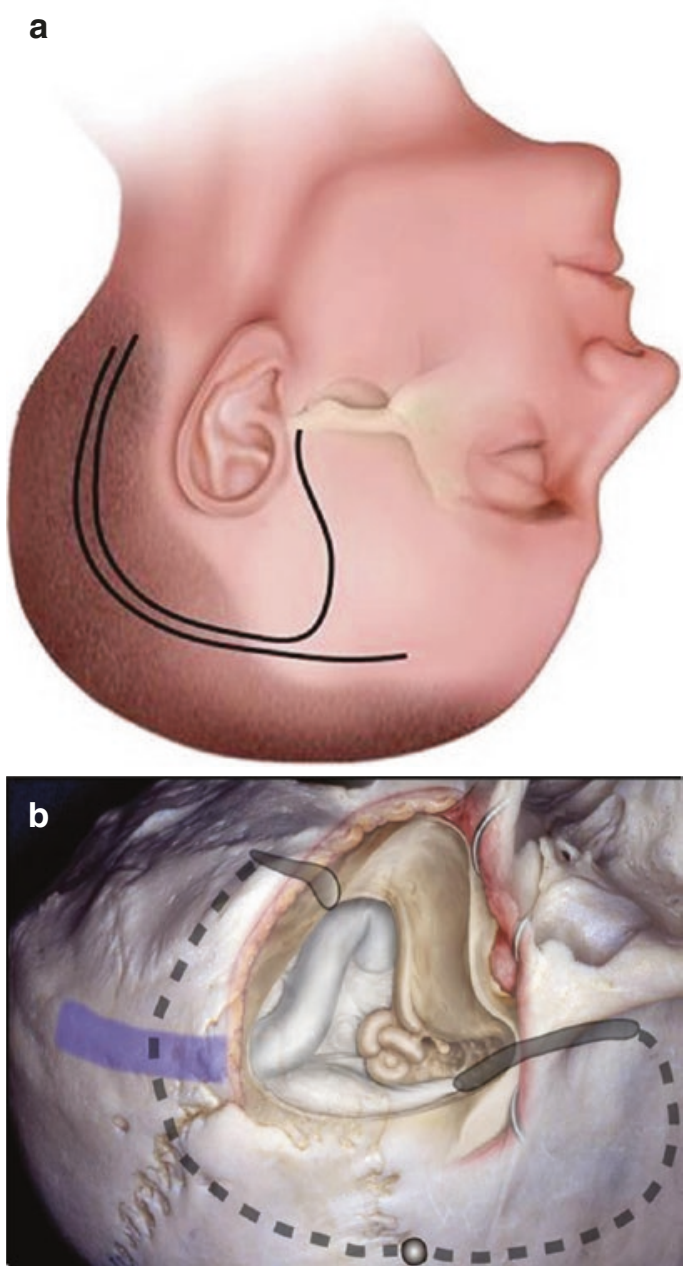

Fig. 8.4 (a) Head position and skin incision for combined transpetrosal approach. (b) The virtual computer image, showing the transverse sigmoid junction until

\section{References}

1. Fukushima T, Sameshima T, Friedman A. Manual of skull base dissection. 2nd ed. Raleigh: AF Neurovideo; 2006.

2. Sameshima T, Mastronardi L, Friedman A, Fukushima T, editors. Fukushima's microanatomy and dissection of the temporal bone for surgery of acoustic neuroma,

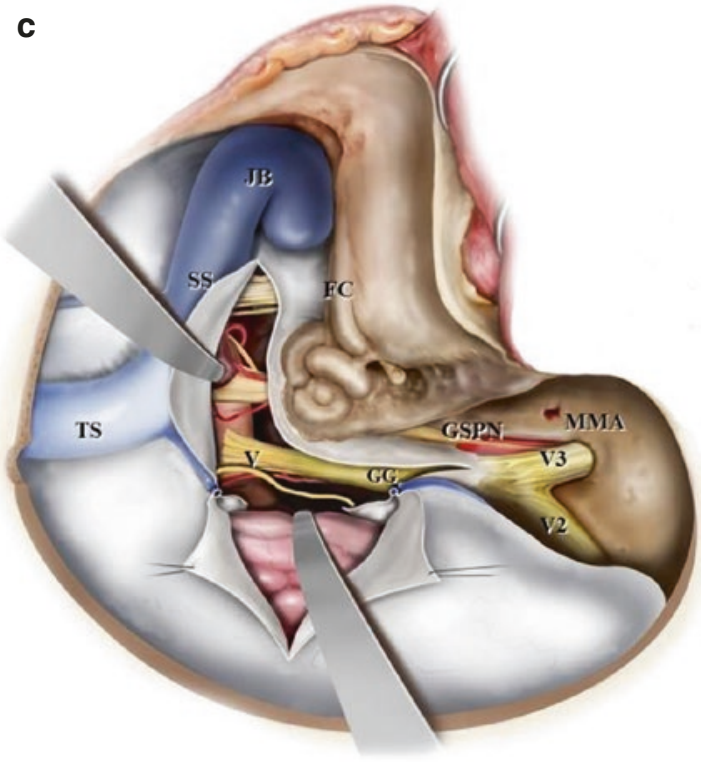

jugular vein, in the relationship with the labyrinth. (c) The dural opening and neurovascular structure

and petroclival meningioma. 2nd ed. Raleigh: AF Neurovideo; 2007. p. 84-114.

3. Tubbs RS, Griessenauer C, Loukas M, Ansari SF, Fritsch MH, Cohen-Gadol AA. Trautmann's triangle anatomy with application to posterior transpetrosal and other related skull base procedures. Clin Anat. 2014;27(7):994-8.

4. Aslan A, Mutlu C, Celik O, Govsa F, Ozgur T, Egrilmez M. Surgical implications of anatomical landmarks on the lateral surface of the mastoid bone. Surg Radiol Anat. 2004;26(4):263-7. 
Open Access This chapter is licensed under the terms of the Creative Commons Attribution 4.0 International License (http://creativecommons.org/licenses/by/4.0/), which permits use, sharing, adaptation, distribution and reproduction in any medium or format, as long as you give appropriate credit to the original author(s) and the source, provide a link to the Creative Commons license and indicate if changes were made.

The images or other third party material in this chapter are included in the chapter's Creative Commons license, unless indicated otherwise in a credit line to the material. If material is not included in the chapter's Creative Commons license and your intended use is not permitted by statutory regulation or exceeds the permitted use, you will need to obtain permission directly from the copyright holder.

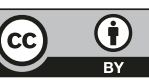

